\title{
Miscellaneous Applications of Quons
}

\author{
Maurice R. KIBLER
}

Université de Lyon, Institut de Physique Nucléaire, Université Lyon 1 and CNRS/IN2P3, 43 bd du 11 novembre 1918, F-69622 Villeurbanne Cedex, France

E-mail: m.kibler@ipnl.in2p3.fr

Received July 23, 2007, in final form September 21, 2007; Published online September 24, 2007

Original article is available at http://www.emis.de/journals/SIGMA/2007/092/

\begin{abstract}
This paper deals with quon algebras or deformed oscillator algebras, for which the deformation parameter is a root of unity. We motivate why such algebras are interesting for fractional supersymmetric quantum mechanics, angular momentum theory and quantum information. More precisely, quon algebras are used for (i) a realization of a generalized Weyl-Heisenberg algebra from which it is possible to associate a fractional supersymmetric dynamical system, (ii) a polar decomposition of $\mathrm{SU}_{2}$ and (iii) a construction of mutually unbiased bases in Hilbert spaces of prime dimension. We also briefly discuss (symmetric informationally complete) positive operator valued measures in the spirit of (iii).
\end{abstract}

Key words: quon algebra; $q$-deformed oscillator algebra; fractional supersymmetric quantum mechanics; polar decompostion of $\mathrm{SU}_{2}$; mutually unbiased bases; positive operator valued measures

2000 Mathematics Subject Classification: 81R50; 81R05; 81R10; 81R15

\section{Introduction}

Deformed oscillator algebras are spanned by generalized bosons or fermions which are often referred to as quons, $q$-bosons, parafermions (parabosons) or $k$-fermions $[1,2,3,4,5,6,7,8,9$, 10]. Such objects are of paramount importance for generating $q$-deformed Lie algebras. In this respect, quons can be used to derive $q$-deformed Lie (super)algebras associated with $q$-deformed models in atomic, molecular and condensed matter physics as well as in nuclear and particle physics.

In recent years, the use of deformed oscillator algebras proved to be useful for many applications in quantum mechanics. For instance, one- and two-parameter deformations of oscillator algebras and Lie algebras were applied to intermediate statistics $[11,12,13,14,15,16,17,18$, 19, 20, 21, 22, 23] and to molecular and nuclear physics [24, 25, 26, 27, 28, 29, 30, 31, 32, 33]. Along this vein, it was shown recently that $q$-bosons play a central role for quantum integrable systems [34].

It is the aim of the present work to motivate why quons, when $q$ is a $k$ th primitive root of unity, are interesting for fractional supersymmetric quantum mechanics of order $k$ and for the determination of mutually unbiased bases used in quantum information. This paper takes it origin in an invited talk at the $3^{\text {rd }}$ International Microconference "Analytic and Algebraic Methods in Physics". It has review character although it exhibits some original aspects partly presented in schools and conferences.

The organisation of the paper is as follows. Section 2 is devoted to some generalities on quon algebras and $k$-fermions which are objects interpolating between bosons and fermions. Section 3 deals with fractional supersymmetric quantum mechanics of order $k$. In Section 4 we show how to construct the Lie algebra of $\mathrm{SU}_{2}$ from two quon algebras. This leads to a polar

${ }^{\star}$ This paper is a contribution to the Proceedings of the 3-rd Microconference "Analytic and Algebraic Methods III". The full collection is available at http://www.emis.de/journals/SIGMA/Prague2007.html 
decomposition which is used in Section 5 for generating mutually unbiased bases (MUBs) in $d$-dimensional Hilbert spaces with $d$ prime. The approach followed for MUBs is also suggested for deriving certain positive operator valued measures (POVMs) in finite-dimensional Hilbert spaces.

Regarding the notations, let us mention that $\delta_{a, b}$ stands for the Kronecker symbol for $a$ and $b$, the bar indicates complex conjugation, $A^{\dagger}$ denotes the adjoint of the operator $A$, I is the identity operator, and $[A, B]_{q}:=A B-q A B$ so that $[A, B]_{1}$ (respectively $[A, B]_{-1}$ ) is the commutator $[A, B]$ (respectively anticommutator $\{A, B\}$ ) of the operators $A$ and $B$.

\section{Quons and $k$-fermions}

We first define a quon algebra or $q$-deformed oscillator algebra for $q$ a root of unity.

Definition 1. The three operators $a_{-}, a_{+}$and $N_{a}$ such that

$$
\left[a_{-}, a_{+}\right]_{q}=\mathbb{I}, \quad\left[N_{a}, a_{ \pm}\right]= \pm a_{ \pm}, \quad a_{ \pm}^{k}=0, \quad N_{a}^{\dagger}=N_{a},
$$

where

$$
q:=\exp (2 \pi i / k), \quad k \in \mathbb{N} \backslash\{0,1\},
$$

define a quon algebra or $q$-deformed oscillator algebra denoted as $A_{q}\left(a_{-}, a_{+}, N_{a}\right)$. The operators $a_{-}$and $a_{+}$are referred to as quon operators. The operators $a_{-}, a_{+}$and $N_{a}$ are called annihilation, creation and number operators, respectively.

Definition 1 differs from the one by Arik and Coon [1] in the sense that we take $q \in S^{1}$ instead of $0<q<1$. For $k=2$ (respectively $k \rightarrow \infty$ ), the quon operators coincide with the ordinary fermion (respectively boson) operators.

For arbitrary $k$, the quon operators $a_{-}$and $a_{+}$are not connected via Hermitian conjugation. It is only for $k=2$ or $k \rightarrow \infty$ that we may take $a_{+}=a_{-}^{\dagger}$. In general (i.e., for $k \neq 2$ or $k \nrightarrow \infty$ ), we have $a_{ \pm}^{\dagger} \neq a_{\mp}$. Therefore, it is natural to consider the so-called $k$-fermionic algebra $\Sigma_{q}$ with the generators $a_{-}, a_{+}, a_{+}^{+}:=a_{+}^{\dagger}, a_{-}^{+}:=a_{-}^{\dagger}$, and $N_{a}[9,10]$. The defining relations for $\Sigma_{q}$ correspond to the ones of $A_{q}\left(a_{-}, a_{+}, N_{a}\right)$ and $A_{\bar{q}}\left(a_{+}^{+}, a_{-}^{+}, N_{a}\right)$ complemented by the relation

$$
a_{-} a_{+}^{+}-q^{-\frac{1}{2}} a_{+}^{+} a_{-}=0 \quad \Leftrightarrow \quad a_{+} a_{-}^{+}-q^{\frac{1}{2}} a_{-}^{+} a_{+}=0 .
$$

Observe that for $k=2$ or $k \rightarrow \infty$, the latter relation corresponds to an identity. The operators $a_{-}, a_{+}, a_{+}^{+}$and $a_{-}^{+}$are called $k$-fermion operators and we also use the terminology $k$-fermions in analogy with fermions and bosons [9]. They clearly interpolate between fermions and bosons.

In a way similar to the one used for ordinary fermions and ordinary bosons, we can define the coherence factor $g^{(m)}$ for an assembly of $m k$-fermions. Such a definition reads

$$
g^{(m)}:=\frac{\left\langle\left(a_{-}^{+}\right)^{m}\left(a_{-}\right)^{m}\right\rangle}{\left\langle a_{-}^{+} a_{-}\right\rangle^{m}},
$$

where

$$
\langle X\rangle:=\frac{(z|X| z)}{(z \mid z)}
$$

stands for the average value of the operator $X$ on the $k$-fermionic coherent state $\mid z)$ defined in [10]. A simple calculation in $\Sigma_{q}$ shows that

$$
\left|g^{(m)}\right|= \begin{cases}0 & \text { for } m>k-1 \\ 1 & \text { for } m \leq k-1 .\end{cases}
$$


From equation (3), we see once again that $k=2$ corresponds to ordinary fermions and $k \rightarrow \infty$ to ordinary bosons. Equation (3) is in agreement with a generalized Pauli exclusion principle according to which, for a many-particle system, a $k$-fermionic state (corresponding to a spin $1 / k$ ) cannot be occupied by more than $k-1$ identical $k$-fermions [9].

To close this section, let us mention that the $k$-fermions introduced in $[9,10]$ share some common properties with the parafermions of order $k-1$ discussed in [35, 36, 37, 38, 39, 40, 41]. Indeed, a parafermionic algebra of order $k-1$ corresponds to a fractional supersymmetric algebra of order $k$.

\section{Quons and fractional supersymmetry}

\subsection{Fractional supersymmetric system}

Following Rubakov and Spiridonov [35], who initially considered the case $k=3$, we start with the definition of a fractional supersymmetric system of order $k$ [42].

Definition 2. For fixed $k$ in $\mathbb{N} \backslash\{0,1\}$, a fractional supersymmetric quantum system of order $k$, or $k$-SUSY system in short, is a doublet $(H, Q)_{k}$ of linear operators $H$ and $Q$, acting on a separable Hilbert space $\mathcal{H}$, such that $H$ is self-adjoint and

$$
Q_{-}:=Q, \quad Q_{+}:=Q^{\dagger}, \quad Q_{ \pm}^{k}=0, \quad \sum_{s=0}^{k-1} Q_{-}^{k-1-s} Q_{+} Q_{-}^{s}=Q_{-}^{k-2} H, \quad\left[H, Q_{ \pm}\right]=0 .
$$

The operators $H$ and $Q_{ \pm}$are called the Hamiltonian and the supercharges of the $k$-SUSY system.

By way of illustration, let us show that the case $k=2$ corresponds to Witten's approach of ordinary supersymmetric quantum mechanics. According to Witten [43], a triplet of linear operators $(H, P, S)$ of linear operators $H, P$ and $S$, with $P$ bounded, defines a supersymmetric quantum system if

$$
S=S^{\dagger}, \quad H=S^{2}, \quad\{S, P\}=0, \quad P^{2}=\mathbb{I}, \quad P=P^{\dagger} .
$$

By putting

$$
Q_{ \pm}=\frac{1}{2} S(1 \pm P)
$$

we get the relations

$$
Q_{+}=Q_{-}^{\dagger}, \quad Q_{ \pm}^{2}=0, \quad\left\{Q_{-}, Q_{+}\right\}=H, \quad\left[H, Q_{ \pm}\right]=0, \quad H=H^{\dagger},
$$

which correspond to a $(H, Q)_{2}$ system or ordinary supersymmetric system with a $Z_{2}$-grading involving fermionic and bosonic states.

Going back to the general case, the doublet $(H, Q)_{k}$, with arbitrary $k$, defines a $k$-SUSY system, with a $Z_{k}$-grading, for which the Hamiltonian $H$ and the two (nonindependent) supercharges $Q_{ \pm}$are up to now formal operators. A natural question arises: How to find realizations of $H$ and $Q_{ \pm}$? In this respect, the definition of a generalized Weyl-Heisenberg algebra is essential [42].

\subsection{Generalized Weyl-Heisenberg algebra}

Definition 3. Let $W_{k}(f)$, where $f=\left\{f_{s}: s=0,1, \ldots, k-1\right\}$ is a set of $k$ functions, be the algebra spanned by the four linear operators $X_{+}, X_{-}, N$ and $K$ acting on the space $\mathcal{H}$ and satisfying

$$
X_{+}=X_{-}^{\dagger}, \quad N=N^{\dagger}, \quad K K^{\dagger}=K^{\dagger} K=\mathbb{I}, \quad K^{k}=\mathbb{I}
$$


and

$$
\begin{aligned}
& {\left[X_{-}, X_{+}\right]=\sum_{s=0}^{k-1} f_{s}(N) \Pi_{s}, \quad \Pi_{s}=\frac{1}{k} \sum_{t=0}^{k-1} q^{-s t} K^{t},} \\
& {\left[N, X_{ \pm}\right]= \pm X_{ \pm}, \quad\left[K, X_{ \pm}\right]_{q^{ \pm 1}}=0, \quad[K, N]=0,}
\end{aligned}
$$

where $q:=\exp (2 \pi i / k)$ and $k \in \mathbb{N} \backslash\{0,1\}$.

It should be realized that, for fixed $k$, equations (4)-(6) define indeed a family of generalized Weyl-Heisenberg algebras $W_{k}(f)$. The various members of the family are distinguished by the various sets $f$.

It is possible to find a realization of $W_{k}(f)$ in terms of one pair $\left(f_{-}, f_{+}\right)$of $k$-fermions with

$$
\left[f_{-}, f_{+}\right]_{q}=\mathbb{I}, \quad f_{ \pm}^{k}=0
$$

and $k$ pairs $\left(b(s)_{-}, b(s)_{+}\right)$of generalized bosons with

$$
\left[b(s)_{-}, b(s)_{+}\right]=f_{s}(N), \quad s=0,1, \ldots, k-1,
$$

such that any $k$-fermion operator commutes with any generalized boson operator. Indeed, by introducing

$$
b_{ \pm}=\sum_{s=0}^{k-1} b(s)_{ \pm} \Pi_{s}
$$

one can take

$$
X_{-}=b_{-}\left(f_{-}+\frac{f_{+}^{k-1}}{[k-1]_{q} !}\right), \quad X_{+}=b_{+}\left(f_{-}+\frac{f_{+}^{k-1}}{[k-1]_{q} !}\right)^{k-1}, \quad K=\left[f_{-}, f_{+}\right],
$$

where

$$
\forall n \in \mathbb{N}^{*}: \quad[n]_{q}:=\frac{1-q^{n}}{1-q}, \quad[n]_{q} !:=[1]_{q}[2]_{q} \cdots[n]_{q} .
$$

\subsection{Realizations of $k$-SUSY systems}

As an important result, the following proposition shows that, for fixed $k$ and given $f$, it is possible to associate a $k$-SUSY system, characterized by a specific doublet $(H, Q)_{k}$, with the algebra $W_{k}(f)$.

Proposition 1. For a fixed value of $k$ and a given set $f$, the relations

$$
Q_{-}:=Q=X_{-}\left(1-\Pi_{1}\right), \quad Q_{+}:=Q^{\dagger}=X_{+}\left(1-\Pi_{0}\right)
$$

and

$$
H=(k-1) X_{+} X_{-}-\sum_{s=3}^{k} \sum_{t=2}^{s-1}(t-1) f_{t}(N-s+t) \Pi_{s}-\sum_{s=1}^{k-1} \sum_{t=s}^{k-1}(t-k) f_{t}(N-s+t) \Pi_{s}
$$

generate a $(H, Q)_{k}$ system associated with the generalized Weyl-Heisenberg algebra $W_{k}(f)$. In addition, the Hamiltonian $H$ can be decomposed as

$$
H=\sum_{s=0}^{k-1} H_{k-s} \Pi_{k-s}
$$


where the various operators $H_{k-s}$ are isospectral Hamiltonians. Each Hamiltonian $H_{k-s}$ acts on a subspace $\mathcal{H}_{k-s}$ of the $Z_{k}$-graded Hilbert space

$$
\mathcal{H}=\bigoplus_{s=0}^{k-1} \mathcal{H}_{k-s}
$$

with $H_{k} \equiv H_{0}$.

In the light of Proposition 1, we foresee that a $k$-SUSY system with a $Z_{k}$-grading can be considered as a superposition of $k-1$ ordinary supersymmetric subsystems (corresponding to $k=2)$ with a $Z_{2}$-grading [42].

As an example, we consider the case of the $Z_{3}$-graded supersymmetric oscillator corresponding to

$$
k=3 \Rightarrow q=\exp \left(\frac{2 \pi i}{3}\right)
$$

with

$$
f_{s}(N)=1, \quad s=0,1,2, \Rightarrow\left[X_{-}, X_{+}\right]=\mathbb{I} .
$$

The corresponding algebra $W_{3}(f)$ can be represented by

$$
\begin{aligned}
& X_{-}=b_{-}\left(f_{-}+\frac{f_{+}^{2}}{[2]_{q} !}\right), \quad X_{+}=b_{+}\left(f_{-}+\frac{f_{+}^{2}}{[2]_{q} !}\right)^{2}, \\
& K=f_{-} f_{+}-f_{+} f_{-}, \quad N=b_{+} b_{-},
\end{aligned}
$$

in terms of 3 -fermions $\left(f_{-}, f_{+}\right)$and ordinary bosons $\left(b_{-}, b_{+}\right)$. The system $(H, Q)_{3}$ associated with $W_{3}(f)$ is defined by

$$
Q_{-}:=Q=X_{-}\left(\Pi_{0}+\Pi_{2}\right), \quad Q_{+}:=Q^{\dagger}=X_{+}\left(\Pi_{1}+\Pi_{2}\right)
$$

and

$$
H=\left(2 X_{+} X_{-}-1\right) \Pi_{3}+\left(2 X_{+} X_{-}+1\right) \Pi_{2}+\left(2 X_{+} X_{-}+3\right) \Pi_{1},
$$

where

$$
\begin{array}{ll}
\Pi_{0} & =\frac{1}{3}\left(1+q^{3} K+q^{3} K^{2}\right), \quad \Pi_{1}=\frac{1}{3}\left(1+q^{1} K+q^{2} K^{2}\right), \\
\Pi_{2} & =\frac{1}{3}\left(1+q^{2} K+q^{1} K^{2}\right),
\end{array}
$$

with $\Pi_{3} \equiv \Pi_{0}$. In terms of 3 -fermions and ordinary bosons, we have

$$
Q_{-}=b_{-} f_{+}\left(f_{-}^{2}-q f_{+}\right), \quad Q_{+}=b_{+}\left(f_{-}-q f_{+}^{2}\right) f_{-}
$$

and

$$
H=2 b_{+} b_{-}-1+2(1-2 q) f_{+} f_{-}+2(1+2 q) f_{+} f_{-} f_{+} f_{-} .
$$

Finally, the energy spectrum of $H$ reads

$$
\operatorname{spectrum}(H)=1 \oplus 2 \oplus 3 \oplus 3 \oplus \cdots,
$$


a symbolic writing to mean that it contains equally spaced levels with a nondegenerate ground state (denoted as 1), a doubly degenerate first excited state (denoted as 2) and an infinite sequence of triply degenerate excited states (denoted as 3 ).

Other sets $f$ lead to other fractional supersymmetric dynamical systems [44]. For instance, the case

$$
f_{s}(N)=a N+b, \quad s=0,1, \ldots, k-1, \quad a \in \mathbb{R}, \quad b \in \mathbb{R}
$$

corresponds to translational shape-invariant systems as for example the harmonic oscillator system (for $a=0$ and $b>0$ ), the Morse system (for $a<0$ and $b>0$ ) and the Pöschl-Teller system (for $a>0$ and $b>0$ ). Furthermore, the case

$$
f_{s}(N)=f_{s}, \quad s=0,1, \ldots, k-1
$$

corresponds to cyclic shape-invariant systems like the Calogero-Vasiliev system for $k=2, f_{0}=$ $1+c$ and $f_{1}=1-c$ with $c \in \mathbb{R}$.

\section{Quons and polar decomposition}

The approach presented in this section is a review based on the original developments given in $[45,46,47,48,49]$. Here, we shall limit ourselves to those aspects which are relevant for Section 5 .

We start with two commuting quon algebras $A_{q}\left(a_{-}, a_{+}, N_{a}\right) \equiv A_{q}(a)$ with $a=x, y$ corresponding to the same value of the deformation parameter $q$. Their generators satisfy equations (1) and (2) with $a=x, y$ and $[X, Y]=0$ for any $X$ in $A_{q}(x)$ and any $Y$ in $A_{q}(y)$. Then, let us look for Hilbertian representations of $A_{q}(x)$ and $A_{q}(y)$ on $k$-dimensional Hilbert spaces $\mathcal{F}_{x}$ and $\mathcal{F}_{y}$ spanned by the orthonormal bases $\left.\left\{\mid n_{1}\right): n_{1}=0,1, \ldots, k-1\right\}$ and $\left.\left\{\mid n_{2}\right): n_{2}=0,1, \ldots, k-1\right\}$, respectively. We easily obtain the representations defined by

$$
\begin{aligned}
& \left.\left.\left.\left.\left.x_{+} \mid n_{1}\right)=\mid n_{1}+1\right), \quad x_{+} \mid k-1\right)=0, \quad x_{-} \mid n_{1}\right)=\left[n_{1}\right]_{q} \mid n_{1}-1\right), \\
& \left.\left.\left.x_{-} \mid 0\right)=0, \quad N_{x} \mid n_{1}\right)=n_{1} \mid n_{1}\right)
\end{aligned}
$$

and

$$
\begin{aligned}
& \left.\left.\left.\left.\left.y_{+} \mid n_{2}\right)=\left[n_{2}+1\right]_{q} \mid n_{2}+1\right), \quad y_{+} \mid k-1\right)=0, \quad y_{-} \mid n_{2}\right)=\mid n_{2}-1\right), \\
& \left.\left.\left.y_{-} \mid 0\right)=0, \quad N_{y} \mid n_{2}\right)=n_{2} \mid n_{2}\right),
\end{aligned}
$$

for $A_{q}(x)$ and $A_{q}(y)$, respectively.

The cornerstone of this approach is to define the two linear operators

$$
h:=\sqrt{N_{x}\left(N_{y}+1\right)}, \quad v_{r a}:=s_{x} s_{y},
$$

with

$$
\begin{aligned}
& s_{x}=q^{a\left(N_{x}+N_{y}\right) / 2} x_{+}+e^{i \phi_{r} / 2} \frac{1}{[k-1]_{q} !}\left(x_{-}\right)^{k-1}, \\
& s_{y}=y_{-} q^{-a\left(N_{x}-N_{y}\right) / 2}+e^{i \phi_{r} / 2} \frac{1}{[k-1]_{q} !}\left(y_{+}\right)^{k-1},
\end{aligned}
$$

where

$$
a \in \mathbb{R}, \quad \phi_{r}=\pi(k-1) r, \quad r \in \mathbb{R} .
$$


The operators $h$ and $v_{r a}$ act on the states

$$
\left.\left.\left.\mid n_{1}, n_{2}\right)=\mid n_{1}\right) \otimes \mid n_{2}\right)
$$

of the $k^{2}$-dimensional space $\mathcal{F}_{x} \otimes \mathcal{F}_{y}$.

We now adapt the trick used by Schwinger in his approach to angular momentum via a coupled pair of harmonic oscillators. This can be done by introducing two new quantum numbers $J$ and $M$ defined by

$$
\left.\left.J:=\frac{1}{2}\left(n_{1}+n_{2}\right), \quad M:=\frac{1}{2}\left(n_{1}-n_{2}\right) \quad \Rightarrow \quad|J M\rangle:=\mid J+M, J-M\right)=\mid n_{1}, n_{2}\right)
$$

Note that

$$
j:=\frac{1}{2}(k-1)
$$

is an admissible value for $J$. Then, let us consider the ( $k$-dimensional) subspace $\epsilon(j)$ of the $\left(k^{2}\right.$-dimensional) space $\mathcal{F}_{x} \otimes \mathcal{F}_{y}$ spanned by the basis

$$
S=\{|j, m\rangle: m=-j,-j+1, \ldots, j\} .
$$

We guess that $\epsilon(j)$ is a space of constant angular momentum $j$. As a matter of fact, we can check that $\epsilon(j)$ is stable under $h$ and $v_{r a}$. Moreover, by defining the operators $j_{ \pm}$and $j_{z}$ through

$$
j_{+}:=h v_{r a}, \quad j_{-}:=v_{r a}^{\dagger} h, \quad j_{z}:=\frac{1}{2}\left(h^{2}-v_{r a}^{\dagger} h^{2} v_{r a}\right),
$$

we obtain

$$
\left[j_{z}, j_{ \pm}\right]= \pm j_{ \pm}, \quad\left[j_{+}, j_{-}\right]=2 j_{z}
$$

for any $r$ in $\mathbb{R}$ and any $a$ in $\mathbb{R}$. The latter commutation relations correspond to the Lie algebra of $\mathrm{SU}_{2}$.

We have here a polar decomposition of $j_{ \pm}$. Thus, from two $q$-deformed oscillator algebras we obtained a polar decomposition of the nondeformed Lie algebra of $\mathrm{SU}_{2}$. In addition, the complete set of commuting operators $\left\{j^{2}, j_{z}\right\}$, where $j^{2}$ is the Casimir of $\mathrm{SU}_{2}$, can be replaced by another complete set of commuting operators, namely $\left\{j^{2}, v_{r a}\right\}$, for which we have the following result. It is to be noted that the operators $z$, defined through $z|j, m\rangle=q^{j-m}|j, m\rangle$, and $v_{r a}$ can be used to generate the Pauli group $\mathcal{P}_{2 j+1}$, a subgroup of order $(2 j+1)^{3}$ of the linear group $\operatorname{GL}(2 j+1, \mathbb{C})$ [49].

Proposition 2. For fixed $a, r$ and $j$, the common orthonormalized eigenvectors of the commuting operators $j^{2}$ and $v_{\text {ra }}$ can be taken in the form

$$
|j \alpha ; r a\rangle=\frac{1}{\sqrt{2 j+1}} \sum_{m=-j}^{j} q^{(j+m)(j-m+1) a / 2-j m r+(j+m) \alpha}|j, m\rangle, \quad \alpha=0,1, \ldots, 2 j,
$$

or alternatively

$$
|j \alpha ; r a\rangle \equiv|a \alpha\rangle=\frac{1}{\sqrt{d}} \sum_{k=0}^{d-1} q^{(d-k-1)(k+1) a / 2+j(k-j) r-(k+1) \alpha}|k\rangle, \quad \alpha=0,1, \ldots, 2 j .
$$

after introducing $k:=j-m, d:=2 j+1$, and $|k\rangle:=|j, m\rangle$. 
In other words, for fixed $a, r$ and $j$, the space $\epsilon(j)$ can be spanned by the basis

$$
B_{r a}=\{|j \alpha ; r a\rangle: \alpha=0,1, \ldots, 2 j\} .
$$

The replacement of the spherical basis $S$, adapted to the chain $\mathrm{SO}_{3} \supset \mathrm{SO}_{2}$ and to the set $\left\{j^{2}, j_{z}\right\}$, by the basis $B_{r a}$, adapted to the chain $\mathrm{SO}_{3} \supset Z_{2 j+1}$ and to the set $\left\{j^{2}, v_{r a}\right\}$, leads to a new form of the Wigner-Racah algebra of $\mathrm{SU}_{2}$ [46]. Note that the notation in (8) is particularly appropriate for quantum information theory (the qudits $|0\rangle,|1\rangle, \ldots,|d-1\rangle$ in the expansion (8) constitute the computational or canonical basis of a $d$-dimensional Hilbert space).

We shall see in Section 5 how the parameter $a$ can be chosen in such a way to generate MUBs.

\section{$5 \quad$ MUBs and POVMs}

\section{$5.1 \quad$ MUBs}

We are now turn to the application of Proposition 2 to the determination of MUBs in a finitedimensional Hilbert space. Let us recall that two orthonormalized bases $\{|a \alpha\rangle: \alpha=0,1, \ldots$, $d-1\}$ and $\{|b \beta\rangle: \beta=0,1, \ldots, d-1\}$ of the $d$-dimensional Hilbert space $\mathbb{C}^{d}$, with an inner product denoted as $\langle\mid\rangle$, are said to be mutually unbiased if $|\langle a \alpha \mid b \beta\rangle|=1 / \sqrt{d}$ for $a \neq b$. In other words, we have

$$
|\langle a \alpha \mid b \beta\rangle|=\delta_{\alpha, \beta} \delta_{a, b}+\frac{1}{\sqrt{d}}\left(1-\delta_{a, b}\right) .
$$

We know that the number of MUBs in $\mathbb{C}^{d}$ is lesser or equal to $d+1$ and that the limit $d+1$ is reached when $d$ is a power of a prime $[50,51,52,53,54,55,56,57,58,59]$.

Let us suppose that $d$ is the power of a prime. Then, we identify $\mathbb{C}^{d}$ with the space $\epsilon(j)$ corresponding to the angular momentum $j=(d-1) / 2$. We begin by introducing the operators

$$
\Pi_{a \alpha}:=|a \alpha\rangle\langle a \alpha|, \quad a=0,1, \ldots, 2 j+1, \quad \alpha=0,1, \ldots, 2 j .
$$

Each of the operators $\Pi_{a \alpha}$ acting on $\mathbb{C}^{d}$ can be considered as a vector in $\mathbb{C}^{d^{2}}$ endowed with the Hilbert-Schmidt inner product. Thus, $\Pi_{a \alpha}$ can be developed as

$$
\Pi_{a \alpha}=\sum_{m=-j}^{j} \sum_{m^{\prime}=-j}^{j} g_{m m^{\prime}}(a \alpha) E_{m m^{\prime}}
$$

where

$$
E_{m m^{\prime}}=|j, m\rangle\left\langle j, m^{\prime}\right|
$$

The calculation of $\operatorname{Tr}\left(\Pi_{a \alpha}^{\dagger} \Pi_{b \beta}\right)$ leads to

$$
\sum_{m=-j}^{j} \sum_{m^{\prime}=-j}^{j} \overline{g_{m m^{\prime}}(a \alpha)} g_{m m^{\prime}}(b \beta)=\delta_{\alpha, \beta} \delta_{a, b}+\frac{1}{d}\left(1-\delta_{a, b}\right) .
$$

By defining the vectors

$$
s(a \alpha):=\left(s_{1}(a \alpha), s_{2}(a \alpha), \ldots, s_{d^{2}}(a \alpha)\right)
$$

with

$$
s_{i}(a \alpha):=g_{m m^{\prime}}(a \alpha), \quad i=(j+m)(2 j+1)+j+m^{\prime}+1,
$$


we get

$$
s(a \alpha) \cdot s(b \beta)=\delta_{\alpha, \beta} \delta_{a, b}+\frac{1}{d}\left(1-\delta_{a, b}\right),
$$

where $s(a \alpha) \cdot s(b \beta)$ stands for the usual inner product $\sum_{i=1}^{d^{2}} \overline{s_{i}(a \alpha)} s_{i}(b \beta)$ in $\mathbb{C}^{d^{2}}$. Note that the relation (12) is independent of the basis chosen for developing the operators $\Pi_{a \alpha}$.

As a result, the determination of the $d(d+1)$ vectors $|a \alpha\rangle$ in $\mathbb{C}^{d}$, for $d$ a power of a prime, amounts to find $d(d+1)$ operators $\Pi_{a \alpha}$ acting on $\mathbb{C}^{d}$ or to find $d(d+1)$ vectors $s(a \alpha)$ in $\mathbb{C}^{d^{2}}$ satisfying (12).

We may now use Proposition 2 derived from the quonic approach of Section 4 to obtain the following result.

Proposition 3. For fixed $r$ and $j=(d-1) / 2$ with $d$ prime, the $d^{2}$ vectors

$$
|a \alpha\rangle:=|j \alpha ; r a\rangle, \quad a=0,1, \ldots, d-1, \quad \alpha=0,1, \ldots, d-1,
$$

where $|j \alpha ; r a\rangle$ is given by (7) or (8) with $q:=\exp (2 \pi i / d)$, together with the $d$ vectors

$$
|a \alpha\rangle:=|j, m\rangle, \quad a=d, \quad \alpha=j+m, \quad m=-j,-j+1, \ldots, j,
$$

constitute a complete set of $d+1$ MUBs in $\mathbb{C}^{d}$.

Proof. From (9), (10) and (11), we get

$$
g_{m m^{\prime}}(a \alpha)=\langle j, m \mid a \alpha\rangle\left\langle a \alpha \mid j, m^{\prime}\right\rangle .
$$

Let us first consider the case $a=0,1, \ldots, d-1$. Equation (7) leads to

$$
\langle j, m \mid a \alpha\rangle=\frac{1}{\sqrt{2 j+1}} q^{(j+m)(j-m+1) a / 2-j m r+(j+m) \alpha} .
$$

The introduction of (13) in

$$
s(a \alpha) \cdot s(b \beta)=\sum_{m=-j}^{j} \sum_{m^{\prime}=-j}^{j} \overline{g_{m m^{\prime}}(a \alpha)} g_{m m^{\prime}}(b \beta)
$$

yields

$$
s(a \alpha) \cdot s(b \beta)=\frac{1}{(2 j+1)^{2}} \sum_{m=-j}^{j} \sum_{m^{\prime}=-j}^{j} q^{\left(m-m^{\prime}\right)\left[\left(m+m^{\prime}+1\right)(a-b) / 2-(\alpha-\beta)\right]}
$$

for $a, b=0,1, \ldots, d-1$. By putting $k=j+m$ and $\ell=j+m^{\prime}$, equation (14) can be rewritten as

$$
s(a \alpha) \cdot s(b \beta)=\frac{1}{d^{2}} \sum_{k=0}^{d-1} \sum_{\ell=0}^{d-1} e^{i \pi(k-\ell)[(k+\ell-d)(a-b)-2(\alpha-\beta)] / d} .
$$

In terms of the generalized quadratic Gauss sums [60]

$$
S(u, v, d):=\sum_{k=0}^{d-1} e^{i \pi\left(u k^{2}+v k\right) / d}
$$

we obtain

$$
s(a \alpha) \cdot s(b \beta)=\frac{1}{d^{2}}|S(u, v, d)|^{2}, \quad u=a-b, \quad v=2(\beta-\alpha)+d(b-a) .
$$


The calculation of $S(u, v, d)$, see [49] and [60], leads to

$$
s(a \alpha) \cdot s(b \beta)=\delta_{\alpha, \beta} \delta_{a, b}+\frac{1}{d}\left(1-\delta_{a, b}\right), \quad a, b=0,1, \ldots, d-1, \quad \alpha=0,1, \ldots, d-1,
$$

which proves that the $d$ bases $B_{r a}:=\{|a \alpha\rangle: \alpha=0,1, \ldots, d-1\}$ with $a=0,1, \ldots, d-1$ are mutually unbiased. It is clear from (13) that the bases $B_{r a}$ with $a=0,1, \ldots, d-1$ and the basis $B_{d}:=\{|d \alpha\rangle: \alpha=0,1, \ldots, d-1\}$ are mutually unbiased. This completes the proof of Proposition 3.

\subsection{POVMs}

An approach similar to the one developed for MUBs can be set up for symmetric informationally complete (SIC) POVMs [61, 62, 63, 64, 65, 66, 67, 68]. We shall briefly discuss the starting point for a study of SIC-POVMs along the lines of Section 5.1 (see [69] for more details).

A SIC-POVM in dimension $d$ can be defined as a set of $d^{2}$ nonnegative operators $P_{x}$ acting on $\mathbb{C}^{d}$ and satisfying

$$
\operatorname{Tr}\left(P_{x} P_{y}\right)=\frac{1}{d+1}\left(d \delta_{x, y}+1\right), \quad \frac{1}{d} \sum_{x=1}^{d^{2}} P_{x}=\mathbb{I}, \quad P_{x}=\left|\Phi_{x}\right\rangle\left\langle\Phi_{x}\right| .
$$

Let

$$
P_{x}=\sum_{m=-j}^{j} \sum_{m^{\prime}=-j}^{j} f_{m m^{\prime}}(a \alpha) E_{m m^{\prime}}
$$

be the development of $P_{x}$ in terms of the operators $E_{m m^{\prime}}$ and let

$$
r(x):=\left(r_{1}(x), r_{2}(x), \ldots, r_{d^{2}}(x)\right)
$$

be the vector in $\mathbb{C}^{d^{2}}$ of components

$$
r_{i}(x):=f_{m m^{\prime}}(a \alpha), \quad i=(j+m)(2 j+1)+j+m^{\prime}+1,
$$

It is immediate to show that

$$
r(x) \cdot r(y)=\frac{1}{d+1}\left(d \delta_{x, y}+1\right),
$$

a relation (independent of the basis chosen for developing $P_{x}$ ) to be compared with (12).

The determination of the $d^{2}$ operators $P_{x}$ satisfying (15) amounts to find $d^{2}$ vectors $\left|\Phi_{x}\right\rangle$ in $\mathbb{C}^{d}$ or $d^{2}$ vectors $r(x)$ in $\mathbb{C}^{d^{2}}$ satisfying (16). The search for solutions of (16) is presently under progress.

\section{Open questions}

To close this paper, we would like to address a few questions which arose during the conference. Possible future developments concern (i) a field theory approach to $k$-fermions and their relation to anyons, (ii) the classification, in terms of the sets $f$, of the Weyl-Heisenberg algebras $W_{k}(f)$ that lead to integrable systems, (iii) the passage from fractional supersymmetric quantum mechanics to fractional supersymmetric non-Hermitian quantum mechanics, as for example along the lines of PT-symmetric regularizations [70], (iv) the Wigner-Racah algebra of the group $\mathrm{SU}_{2}$ in the $\left\{j^{2}, v_{r a}\right\}$ scheme, and (v) the construction of MUBs and SIC-POVMs in an unified way. 
Some comments regarding points (iv) and (v) are in order.

The $\left\{j^{2}, v_{r a}\right\}$ scheme described in Section 4 constitutes an alternative to the familiar $\left\{j^{2}, j_{z}\right\}$ scheme of angular momentum theory. As a further step, it would be interesting to find differential realizations of the operator $v_{r a}$ as well as realizations of the bases $B_{r a}$ on the sphere $S^{2}$ for $j$ integer and on Fock-Bargmann spaces (in 1 and 2 dimensions) for $j$ integer or half of an odd integer.

The preliminary study reported in Section 5 for MUBs and POVMs is based on the replacement of

$$
\begin{aligned}
& |\langle a \alpha \mid b \beta\rangle|=\delta_{\alpha, \beta} \delta_{a, b}+\frac{1}{\sqrt{d}}\left(1-\delta_{a, b}\right), \\
& \left|\left\langle\Phi_{x} \mid \Phi_{y}\right\rangle\right|=\frac{1}{\sqrt{d+1}} \sqrt{d \delta_{x, y}+1},
\end{aligned}
$$

corresponding to inner products in $\mathbb{C}^{d}$, by

$$
\begin{aligned}
& s(a \alpha) \cdot s(b \beta)=\delta_{\alpha, \beta} \delta_{a, b}+\frac{1}{d}\left(1-\delta_{a, b}\right), \\
& r(x) \cdot r(y)=\frac{1}{d+1}\left(d \delta_{x, y}+1\right),
\end{aligned}
$$

corresponding to inner products in $\mathbb{C}^{d^{2}}$, respectively. We may ask the question: Is it easier to find solutions of (17), (18) than to find solutions of (19), (20)? We do not have any answer. In the case of MUBs, we have a general solution (see Proposition 3) of (19) and thus (17) for $d$ prime in the framework of angular momentum theory. It would be interesting to extend this result for $d$ a power of a prime. In the case of SIC-POVMs, to prove or disprove the conjecture according to which SIC-POMVs exist in any dimension amounts to prove or disprove that (20) has a solution in any dimension.

Note. After the submission (July 23, 2007) of the present paper for publication in SIGMA, the author became aware of a preprint dealing with the existence of SIC-POVMs posted (July 20, 2007) on arXiv [71]. The main result in [71] is that SIC-POVMs exist in all dimensions. As a corollary of this result, equation (20) admits solutions in any dimension.

\section{Acknowledgements}

Some parts of the material reported here were worked out in collaboration with Mohammed Daoud, Olivier Albouy, and Michel Planat. The present paper is a contribution to the $3^{\text {rd }}$ International Microconference "Analytic and Algebraic Methods in Physics" (June 2007, Villa Lanna, Prague); the author is very indebted to Miloslav Znojil for organizing the conference and for useful comments; thanks are due to Uwe Günther, Stefan Rauch-Wojciechowski, Artur Sergyeyev, Petr Šulcp, and Pierguilio Tempesta for interesting discussions. This work was also presented at the workshop "Finite Projective Geometries in Quantum Theory" (August 2007, Astronomical Institute, Tatranská Lomnica); the author acknowledges the organizer, Metod Saniga, and the other participants for fruitful interactions.

\section{References}

[1] Arik M., Coon D.D., Hilbert spaces of analytic functions and generalized coherent states, J. Math. Phys. 17 (1976), 524-527.

[2] Kuryshkin M.V., Opérateurs quantiques généralisés de création et d'annihilation, Ann. Fond. Louis de Broglie 5 (1980), 111-125. 
[3] Jannussis A.D., Papaloucas L.C., Siafarikas P.D., Eigenfunctions and eigenvalues of the $q$-differential operators, Hadronic J. 3 (1980), 1622-1632.

[4] Biedenharn L.C., The quantum group $\mathrm{SU}_{q}(2)$ and a $q$-analogue of the boson operators, J. Phys. A: Math. Gen. 22 (1989), L873-L878.

[5] Sun C.-P., Fu H.-C., The $q$-deformed boson realisation of the quantum group $\mathrm{SU}(n)_{q}$ and its representations, J. Phys. A: Math. Gen. 22 (1989), L983-L986.

[6] Macfarlane A.J., On $q$-analogues of the quantum harmonic oscillator and the quantum group $\operatorname{SU}(2)_{q}$, J. Phys. A: Math. Gen. 22 (1989), 4581-4588.

[7] Chaturvedi S., Kapoor A.K., Sandhya R., Srinivasan V., Simon R., Generalized commutation relations for a single-mode oscillator, Phys. Rev. A 43 (1991), 4555-4557.

[8] Greenberg O.W., Quons, an interpolation between Bose and Fermi oscillators, cond-mat/9301002.

[9] Daoud M., Hassouni Y., Kibler M., The $k$-fermions as objects interpolating between fermions and bosons, in Symmetries in Science X, Editors B. Gruber and M. Ramek, Plenum Press, New York, 1998, 63-67, quant-ph/9710016.

[10] Daoud M., Hassouni Y., Kibler M., Generalized supercoherent states, Phys. Atom. Nuclei 61 (1998), 18211824, quant-ph/9804046.

[11] Ge M.-L., Su G., The statistical distribution function of the $q$-deformed harmonic-oscillator, J. Phys. A: Math. Gen. 24 (1991), L721-L723.

[12] Martín-Delgado M.A., Planck distribution for a q-boson gas, J. Phys. A: Math. Gen. 24 (1991), L1285L1291.

[13] Lee C.R., Yu J.-P., On q-deformed free-electron gases, Phys. Lett. A 164 (1992), 164-166.

[14] Su G., Ge M.-L., Thermodynamic characteristics of the q-deformed ideal Bose-gas, Phys. Lett. A 173 (1993), $17-20$.

[15] Tuszyński J.A., Rubin J.L, Meyer J., Kibler M., Statistical mechanics of a $q$-deformed boson gaz, Phys. Lett. A 175 (1993), 173-177.

[16] Man'ko V.I., Marmo G., Solimeno S., Zaccaria F., Correlation functions of quantum q-oscillators, Phys. Lett. A 176 (1993), 173-175, hep-th/9303008.

[17] Hsu R.-R., Lee C.-R., Statistical distribution of gases which obey $q$-deformed commutation relations, Phys. Lett. A 180 (1993), 314-316.

[18] Granovskii Ya.I., Zhedanov A.S., Production of $q$-bosons by a classical current - an exactly solvable model, Modern Phys. Lett. A 8 (1993), 1029-1035.

[19] Chaichian M., Felipe R.G., Montonen C., Statistics of $q$-oscillators, quons and relations to fractional statistics, J. Phys. A: Math. Gen. 26 (1993), 4017-4034, hep-th/9304111.

[20] Gupta R.K., Bach C.T., Rosu H., Planck distribution for a complex q-boson gas, J. Phys. A: Math. Gen. 27 (1994), 1427-1433.

[21] R.-Monteiro M.A., Roditi I., Rodrigues L.M.C.S., Gamma-point transition in quantum q-gases, Phys. Lett. A 188 (1994), 11-15.

[22] Gong R.-S., Thermodynamic characteristics of the ( $p, q)$-deformed ideal Bose-gas, Phys. Lett. A 199 (1995), $81-85$.

[23] Daoud M., Kibler M., Statistical-mechanics of $q p$-bosons in D-dimensions, Phys. Lett. A 206 (1995), 13-17, quant-ph/9512006.

[24] Witten E., Gauge-theories, vertex models, and quantum groups, Nuclear Phys. B 330 (1990), $285-346$.

[25] Iwao S., Knot and conformal field-theory approach in molecular and nuclear-physics, Prog. Theor. Phys. 83 (1990), 363-367.

[26] Bonatsos D., Raychev P.P., Roussev R.P., Smirnov Yu.F., Description of rotational molecular-spectra by the quantum algebra $\mathrm{SU}_{q}(2)$, Chem. Phys. Lett. 175 (1990), 300-306.

[27] Chang Z., Guo H.-Y., Yan H., The $q$-deformed oscillator model and the vibrational-spectra of diatomicmolecules, Phys. Lett. A 156 (1991), 192-196.

[28] Chang Z., Yan H., Diatomic-molecular spectrum in view of quantum group-theory, Phys. Rev. A 44 (1991), 7405-7413.

[29] Bonatsos D., Drenska S.B., Raychev P.P., Roussev R.P., Smirnov Yu.F., Description of superdeformed bands by the quantum algebra $\mathrm{SU}_{q}(2)$, J. Phys. G: Nucl. Part. Phys. 17 (1991), L67-L73. 
[30] Bonatsos D., Raychev P.P., Faessler A., Quantum algebraic description of vibrational molecular-spectra, Chem. Phys. Lett. 178 (1991), 221-226.

[31] Bonatsos D., Argyres E.N., Raychev P.P., $\mathrm{SU}_{q}(1,1)$ description of vibrational molecular-spectra, J. Phys. A: Math. Gen. 24 (1991), L403-L408.

[32] Jenkovszky L., Kibler M., Mishchenko A., Two-parameter quantum-deformed dual amplitude, Modern Phys. Lett A 10 (1995), 51-60, hep-th/9407071.

[33] Barbier R., Kibler M., Application of a two-parameter quantum algebra to rotational spectroscopy of nuclei, Rep. Math. Phys. 38 (1996), 221-226, nucl-th/9602015.

[34] Kundu A., q-boson in quantum integrable systems, SIGMA 3 (2007), 040, 14 pages, nlin.SI/0701030.

[35] Rubakov V.A., Spiridonov V.P., Parasupersymmetric quantum-mechanics, Modern Phys. Lett. A 3 (1988), 1337-1347.

[36] Beckers J., Debergh N., Parastatistics and supersymmetry in quantum-mechanics, Nuclear Phys. B 340 (1990), 767-776.

[37] Debergh N., On parasupersymmetric Hamiltonians and vector-mesons in magnetic-fields, J. Phys. A: Math. Gen. 27 (1994), L213-L217.

[38] Khare A., Parasupersymmetry in quantum mechanics, J. Math. Phys. 34 (1993), 1277-1294.

[39] Filippov A.T., Isaev A.P., Kurdikov A.B., Paragrassmann extensions of the Virasoro algebra, Internat. J. Modern Phys. A 8 (1993), 4973-5003, hep-th/9212157.

[40] Durand S., Fractional superspace formulation of generalized mechanics, Modern Phys. Lett. A 8 (1993), 2323-2334, hep-th/9305130.

[41] Klishevich S., Plyushchay T., Supersymmetry of parafermions, Modern Phys. Lett. A 14 (1999), 2739-2752, hep-th/9905149.

[42] Daoud M., Kibler M., Fractional supersymmetric quantum mechanics as a set of replicas of ordinary supersymmetric quantum mechanics, Phys. Lett. A 321 (2004), 147-151, math-ph/0312019.

[43] Witten E., Dynamical breaking of supersymmetry, Nuclear Phys. B 188 (1981), 513-554.

[44] Daoud M., Kibler M.R., Fractional supersymmetry and hierarchy of shape invariant potentials, J. Math. Phys. 47 (2006), 122108, 11 pages, quant-ph/0609017.

[45] Kibler M., Daoud M., Variations on a theme of quons: I. A non standard basis for the Wigner-Racah algebra of the group SU(2), Recent Res. Devel. Quantum Chem. 2 (2001), 91-99, physics/9712034.

[46] Kibler M.R., Representation theory and Wigner-Racah algebra of the group SU(2) in a noncanonical basis, Collect. Czech. Chem. Commun. 70 (2005), 771-796, quant-ph/0504025.

[47] Kibler M.R., Angular momentum and mutually unbiased bases, Internat. J. Modern Phys. B 20 (2006), 1792-1801, quant-ph/0510124.

[48] Kibler M.R., Planat M., A SU(2) recipe for mutually unbiased bases, Internat. J. Modern Phys. B 20 (2006), 1802-1807, quant-ph/0601092.

[49] Albouy O., Kibler M.R., $\mathrm{SU}_{2}$ nonstandard bases: case of mutually unbiased bases, SIGMA 3 (2007), 076, 22 pages, quant-ph/0701230.

[50] Ivanović I.D., Geometrical description of quantum state determination, J. Phys. A: Math. Gen. 14 (1981), 3241-3245.

[51] Šťovíček P., Tolar J., Quantum mechanics in a discrete space-time, Rep. Math. Phys. 20 (1984), 157-170.

[52] Balian R., Itzykson C., Observations sur la mécanique quantique finie, C.R. Acad. Sci. (Paris) 303 (1986), 773-778.

[53] Wootters W.K., Fields B.D., Optimal state-determination by mutually unbiased measurements, Ann. Phys. (N.Y.) 191 (1989), 363-381.

[54] Barnum H., MUBs and spherical 2-designs, quant-ph/0205155.

[55] Bandyopadhyay S., Boykin P.O., Roychowdhury V., Vatan F., A new proof for the existence of mutually unbiased bases, Algorithmica 34 (2002), 512-528, quant-ph/0103162.

[56] Chaturvedi S., Aspects of mutually unbiased bases in odd-prime-power dimensions, Phys. Rev. A 65 (2002), 044301, 3 pages, quant-ph/0109003.

[57] Pittenger A.O., Rubin M.H., Mutually unbiased bases, generalized spin matrices and separability, Linear Algebra Appl. 390 (2004), 255-278, quant-ph/0308142. 
[58] Klappenecker A., Rötteler M., Constructions of mutually unbiased bases, Lect. Notes Comput. Sci. 2948 (2004), 137-144, quant-ph/0309120.

[59] Bengtsson I., Three ways to look at mutually unbiased bases, quant-ph/0610216.

[60] Berndt B.C., Evans R.J., Williams K.S., Gauss and Jacobi Sums, Wiley, New York, 1998.

[61] Zauner G., Quantendesigns: Grundzüge einer nichtcommutativen Designtheorie, Diploma Thesis, University of Wien, 1999.

[62] Caves C.M., Fuchs C.A., Schack R., Unknown quantum states: the quantum de Finetti representation, J. Math. Phys. 43 (2002), 4537-4559, quant-ph/0104088.

[63] Renes J.M., Blume-Kohout R., Scott A.J., Caves C.M., Symmetric informationally complete quantum measurements, J. Math. Phys. 45 (2004), 2171-2180, quant-ph/0310075.

[64] Appleby D.M., Symmetric informationally complete-positive operator valued measures and the extended Clifford group, J. Math. Phys. 46 (2005), 052107, 29 pages, quant-ph/0412001.

[65] Grassl M., Tomography of quantum states in small dimensions, Elec. Notes Discrete Math. 20 (2005), $151-164$.

[66] Klappenecker A., Rötteler M., Mutually unbiased bases are complex projective 2-designs, quant-ph/0502031.

[67] Klappenecker A., Rötteler M., Shparlinski I.E., Winterhof A., On approximately symmetric informationally complete positive operator-valued measures and related systems of quantum states, J. Math. Phys. 46 (2005), 082104, 17 pages, quant-ph/0503239.

[68] Weigert S., Simple minimal informationally complete measurements for qudits, Internat. J. Modern Phys. B 20 (2006), 1942-1955, quant-ph/0508003.

[69] Albouy A., Kibler M.R., A unified approach to SIC-POVMs and MUBs, arXiv:0704.0511.

[70] Znojil M., PT-symmetric regularizations in supersymmetric quantum mechanics, J. Phys. A: Math. Gen. 37 (2004), 10209-10222, hep-th/0404145.

[71] Hall J.L., Rao A., SIC-POVMs exist in all dimensions, arXiv:0707.3002. 